\title{
CIÊNCIANATURA
}

\section{Eventos extremos anuais de precipitação em Mauriti - CE}

Extreme annual precipitation events in Mauriti - CE

\section{Rosa Lindjhenys de Oliveira Jorge e Daisy Beserra lucena}

Graduanda em Engenharia Ambiental, Centro de Tecnologia, Universidade Federal da Paraíba, João Pessoa, PB, Brasil Professora Adjunta do Departamento de Geociências, Universidade Federal da Paraíba, João Pessoa, PB, Brasil

\section{Resumo}

Este trabalho tem como objetivo identificar e determinar a intensidade dos eventos extremos de chuva no município de Mauriti, localizado na região Sul do Estado do Ceará. Os dados de precipitação diária para o posto de Mauritil CE foram obtidos a partir da Fundação Cearense de Meteorologia e Recursos Hídricos - FUNCEME, para o período de 1974 a 2016. Os anos com eventos extremos de precipitação foram identificados utilizando o Índice de Anomalia de Chuva (IAC). De acordo com a classificação das severidades positivas e negativas, 10 anos foram classificados como muito secos, 6 anos como muito chuvosos, 2 como extremamente chuvosos e os demais anos analisados $(58,14 \%)$ apresentaram uma variabilidade natural. Os anos extremos foram analisados levando em consideração a influência que os fenômenos climáticos sobre os oceanos Pacífico e Atlântico exercem sobre estes acontecimentos. Os resultados indicaram que os anos extremos apresentam um grau de relação maior com os eventos sobre o Pacífico (El Niño e La Niña) do que com Dipolo do Atlântico.

Palavras-chave: IAC; ENOS; Dipolo

\section{Abstract}

This work aims to identify and determine the intensity of extreme rainfall events in the municipality of Mauriti, located in the southern region of the state of Ceará. The daily precipitation data for the Mauriti / CE station were obtained from the Cearense Foundation of Meteorology and Water Resources - FUNCEME, for the period from 1974 to 2016. The years with extreme precipitation events were identified using the Anomaly Index of Rainfall (IAC). According to the classification of positive and negative severities, 10 years were classified as very dry, 6 years as very rainy, 2 as extremely rainy and the other years analyzed (58.14\%) presented a natural variability. The extreme years were analyzed taking into account the influence that climatic phenomena on the Pacific and Atlantic oceans exert on these events. The results indicated that the extreme years present a higher degree of relationship with the events on the Pacific (El Niño and La Niña) than with the Atlantic Dipole.

Keywords: RAI; ENSO; Dipole

\footnotetext{
*lindjhenysrosa@hotmail.com
} 


\section{Introdução}

A chuva é um dos elementos climáticos mais importantes para o planejamento territorial a curto e longo prazo, pois afeta de forma sensível os mais variados grupos sociais, assim como instituições públicas, privadas e as dinâmicas da vida social de forma ampla (RAMIRES et al., 2017). A chuva apresenta uma grande variabilidade espacial e temporal em algumas regiões do planeta, como por exemplo, na região semiárida do Nordeste do Brasil (MARENGO et al., 2011). Neste elemento é importante analisar tanto os eventos de chuvas que ocorrem numa faixa habitual (variabilidade esperada), quanto os que são definidos como eventos extremos (secas ou enchentes).

De acordo com Sousa (2010), os eventos extremos são definidos como os totais de chuva em um certo período - seja anual, sazonal ou diário - que apresentam desvios muito superiores ou inferiores ao comportamento habitual da área no período analisado.

Assim, percebe-se que o conhecimento do comportamento das chuvas intensas é de grande valia para o planejamento do uso e ocupação da terra, de forma a prevenir os impactos associado a esses episódios (SOUSA, 2010).

Os eventos extremos de precipitação estão sendo estudados em escala global, nacional e regional (NÓBREGA et al., 2014). No estado do Ceará, localizado ao norte do Nordeste Brasileiro (NEB), vários estudos como os de Costa e Silva (2017) e Santos e Manzi (2010), analisaram a ocorrência de eventos extremos de precipitação, mostrando a relação com as anomalias de temperatura da superfície do mar (ATSM) dos oceanos Pacifico e Atlântico, destacando a influência exercida.

A ocorrência de eventos extremos, como secas severas ou chuvas excessivas, têm sido relacionados aos padrões anômalos de grande escala da circulação atmosférica global associados ao fenômeno El Niño-Oscilação Sul (ENOS) (ANDREOLI et al., 2004). O fenômeno ENOS é caraterizado por anomalias, positivas (El Niño) ou negativas (La Niña), de Temperatura da Superfície do Mar (TSM) no Pacífico Equatorial (CPTEC/INPE, 2018).

De acordo com Reboita e Santos (2014), embora os efeitos do ENOS sejam observacionalmente evidentes, não se deve analisar a precipitação sem levar em consideração o padrão das anomalias da TSM no Atlântico Tropical (AT). O fenômeno El Niño não é sempre sinônimo de seca (NÓBREGA; SANTIAGO, 2014), pois as ocorrências simultâneas de El Niño e secas no NEB, restringem-se a determinados eventos, diante disso a precipitação nessa região tem sido fortemente relacionada as Anomalias de Temperatura de Superfície do Mar no AT, principalmente o evento conhecido como Dipolo do Atlântico (ANDREOLI et al., 2007).

O Dipolo do Atlântico é caracterizado por um gradiente térmico norte-sul e inter-hemisférico que envolve variações da TSM em ambos os hemisférios, em escala sazonal, interanual e decadal (LUCENA, 2008). Apesar das ATSM serem conhecidas historicamente como dipolo do Atlântico, a correlação entre as TSM de ambas as regiões só é significativa, ou seja, fortemente negativa, com uma pequena frequência (12-15\% do tempo) e pode ser resultado da sobreposição de variáveis independentes, ao norte e ao sul da Zona de Convergência Intertropical (ZCIT) (SASAKI, 2014). Por não caracterizar de fato um dipolo, alguns autores preferem não utilizar a nomenclatura de dipolo, e utilizam gradiente inter-hemisférico ou meridional da TSM (GRADM) ou gradiente meridional de Atlântico Tropical (GMAT), já que esse gradiente ocorre com uma frequência maior.

O Dipolo do atlântico pode ser positivo ou negativo. O dipolo positivo caracteriza-se por valores maiores da ATSM do Atlântico Tropical Norte em relação ao Atlântico Tropical Sul, por outro lado, o dipolo negativo acontece quando o Atlântico Tropical Sul apresenta maiores valores de ATSM. Esse padrão de anomalias influencia na posição latitudinal da ZCIT, considerado o sistema mais importante gerador de precipitação sobre a região equatorial do Oceano Atlântico, assim como áreas continentais adjacentes (CORRÊA, 2016)

Diante do exposto, é perceptível que tanto o ENOS quanto o Dipolo do Atlântico exercem influência na precipitação do NEB. Esses fenômenos também podem atuar de forma conjunta, contribuindo para ocorrências de eventos extremos sobre a região. Giannini et al. (2004) mostrou que em anos de ocorrência simultânea do EL Niño (La Niña) e do dipolo positivo (negativo), as condições de precipitação se apresentam, respectivamente, abaixo (acima) do normal para o NEB.

A ocorrência de eventos extremos de precipitação ativa a necessidade de um melhor conhecimento e interpretação das escalas da variabilidade pluviométricas, que podem ser efetuadas por meio de índices (Alves et al., 2016). A utilização do Índice de Anomalia Climática (IAC) tem se revelado uma importante ferramenta de análise das precipitações considerandose sua simplicidade procedimental, os recursos computacionais e a determinação qualitativa de anomalias extremas (Sanches et al., 2014). Vários estudos como os de Alves et al. (2016) e Maniçoba et al. (2017), utilizam o IAC para determinação dos eventos extremos de chuva em diversos estados do NEB, destacando, dentre outros, o de Costa e Silva (2017) que analisou a distribuição espaço-temporal do IAC para o Estado do Ceará. 
O município foco do estudo é Mauriti, localizado na região Sul do estado do Ceará. O município tem como principal atividade econômica a agricultura, sendo o $5^{\circ}$ maior produtor de grãos do estado, destacando-se o milho e o feijão, seguindose por frutas e hortaliças (EMATERCE, 2017).

Diante dos impactos causados pelos eventos extremos de chuvas sobre a agricultura, o conhecimento e interpretação da variabilidade pluviométrica torna-se muito importante, dessa forma, este trabalho tem como objetivo identificar e determinar a intensidade dos eventos extremos de chuvas no município de Mauriti.

\section{Materiais e métodos}

\section{1 Área de estudo}

O município de Mauriti (7²3'21"S, 3846'28"W, $347 \mathrm{~m}$ ) está localizado no Sul do Ceará (Mapa 1), que apresenta clima semiárido quente em grande parte de sua área de abrangência (CARVALHO et al., 2007).

Figura 1 - Localização do município de Mauriti - CE

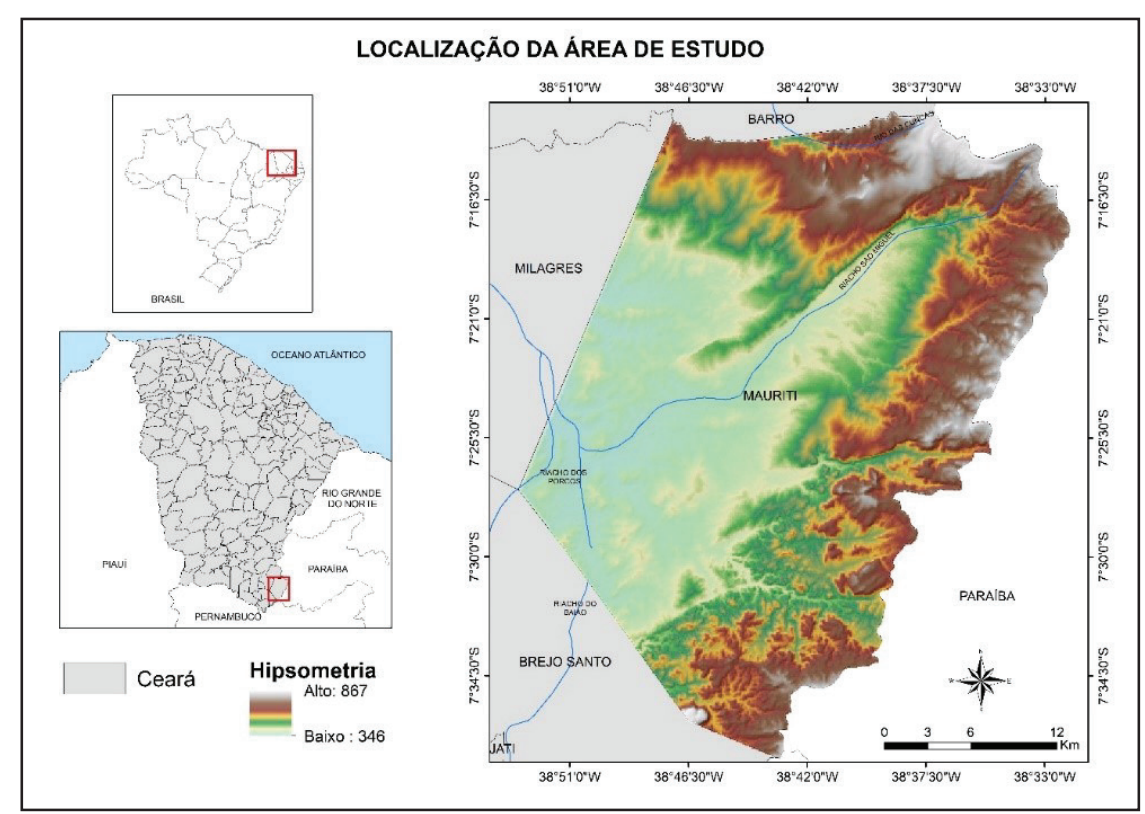

Fonte: Elaborado pelo Autor, 2018

De acordo com o Instituto Brasileiro de Geografia e Estatística (IBGE, 2017), Mauriti possui uma área de 1049,488 $\mathrm{km}^{2}$ e população estimada de 46.548 habitantes. O município faz limite com as cidades Barro (ao Norte), Milagres (a Oeste), e Brejo Santo (a Sul) e com estado da Paraíba (a Leste) (IPECE, 2016).

Como visualizado no Mapa 1, Mauriti apresenta hipsometria variando de $346 \mathrm{~m}$ a $867 \mathrm{~m}$, em sua porção oriental temse os maiores valores de altitude diminuindo para o setor leste.

\subsection{Dados}

Os dados de precipitação diária para o posto de Mauriti/CE foram obtidos a partir da Fundação Cearense de Meteorologia e Recursos Hídricos - FUNCEME, para o período de 1974 a 2016, o que totaliza 43 anos de dados. O preenchimento das falhas existentes na série de dados foi realizado por meio da média diária do dia no mês em questão. Os anos que apresentaram falhas foi o ano de 2010, entre o dia 9 e 31 de outubro, e o ano de 2012, entre o dia 23 e 31 de dezembro. As falhas correspondem apenas a, aproximadamente, $0,20 \%$ dos dados.

Para identificação dos anos de eventos sobre os oceanos Pacífico (ENOS) e Atlântico (Dipolo), utilizou-se como base a relação de dados de temperatura de superfície do mar (TSM) provenientes do Climate Prediction Center (CPC/NOOA), disponível no site http://www.cpc.ncep.noaa.gov/data/indices/sstoi.atl.indices. 


\section{3 Índice de Anomalia de Chuva - IAC}

Para analisar o comportamento da precipitação ao longo do período em estudo, identificando os eventos extremos de precipitação, foi utilizado o Índice de Anomalia de Chuva (IAC), proposto por Rooy (1965), que torna possível a comparação do desvio de precipitação em relação à condição normal de diversas regiões (FREITAS, 2005). O IAC é expresso por:

$I A C=3 *\left[\frac{N-\bar{N}}{\bar{M}-N}\right]$, para anomalias positivas
$I A C=-3 *\left[\frac{N-\bar{N}}{\bar{X}-N}\right]$, para anomalias negativas

Em que:

$\mathrm{N}$ - Precipitação total do ano que se deseja calcular o IAC (mm);

$\bar{N}$ - Precipitação média anual da série histórica $(\mathrm{mm})$;

$\bar{M}$ - Média das dez maiores precipitações mensais da série histórica (mm);

$\bar{X}$ - Média das dez menores precipitações mensais da série histórica (mm).

Além de possibilitar a determinação das anomalias extremas, o IAC torna possível ordena-las de acordo com suas magnitudes (FERNANDES et al., 2009). Para isso, os valores de IAC são dispostos em faixas que classificam as severidades positivas e negativas de acordo com a intensidade (Tabela 1), para tanto, foi utilizada a classificação elaborada por Araújo et al. (2009) para os anos secos e úmidos, com adaptação para anos secos e chuvosos.

Tabela 1 - Classificação do IAC de acordo com a intensidade

\begin{tabular}{l|c}
\hline Faixa de IAC & Classe de intensidade \\
\hline$\geq 4$ & Extremamente chuvoso \\
\hline Entre 2 e 4 & Muito chuvoso \\
\hline Entre 0 e 2 & Chuvoso \\
\hline Entre 0 e -2 & Seco \\
\hline Entre -2 e -4 & Muito Seco \\
\hline$\leq-4$ & Extremamente Seco \\
\hline
\end{tabular}

Fonte: Adaptado de Araújo et al. (2009)

\subsection{Eventos sobre os oceanos}

A seleção dos anos de El Niño e La Niña tiveram como base a relação estabelecida pelo CPC/NOOA, que se baseia em um limiar de $+/-0,5^{\circ} \mathrm{C}$ para o Índice Oceânico de Niño (ONI) [média de 3 meses de anomalias de TSM ERSST.v5 na região de Niño $3.4\left(5^{\circ} \mathrm{N}-5^{\circ} \mathrm{S}, 120^{\circ}-170^{\circ} \mathrm{W}\right)$ ]. Para classificar os eventos nas categorias forte, moderada e fraca, foi utilizada a relação do Golden Gate Weather Services (Tabela 2).

Tabela 2 - Classificação da intensidade do Índice Oceânico de Niño

\begin{tabular}{c|c|c}
\hline \multirow{2}{*}{ Evento } & Índice Oceânico de Niño & Intensidade \\
\hline \multirow{4}{*}{ El Niño } & 0,5 a 0,9 & Fraco \\
\cline { 2 - 3 } & 1,0 a 1,4 & Moderado \\
\cline { 2 - 3 } & 1,5 a 1,9 & Forte \\
\cline { 2 - 3 } & $\geq 2$ & Muiro Forte \\
\hline \multirow{4}{*}{ La Niña } & $-0,5$ a $-0,9$ & Fraca \\
\cline { 2 - 3 } & $-1,0$ a $-1,4$ & Moderada \\
\cline { 2 - 3 } & $-1,5$ a $-1,9$ & Forte \\
\cline { 2 - 3 } & $\leq-2$ & Muito Forte \\
\hline
\end{tabular}


$\mathrm{Na}$ Tabela 3 podem ser visualizados os anos durante o período de 1974-2016 em que ocorreram os eventos El Niño e La Niña e suas respectivas intensidades.

Tabela 3 - Classificação da intensidade do Índice Oceânico de Niño

\begin{tabular}{c|c|c|c|c|c|c}
\hline \multicolumn{5}{c|}{ EI Niño } & \multicolumn{3}{c}{ La Niña } \\
\hline Fraco & Moderado & Forte & Muito Forte & Fraca & Moderada & Forte \\
\hline $76 / 77$ & $86 / 87$ & $87 / 88$ & $82 / 83$ & $74 / 75$ & $95 / 96$ & $73 / 74$ \\
\hline $77 / 78$ & $94 / 95$ & $91 / 92$ & $97 / 98$ & $83 / 84$ & $11 / 12$ & $75 / 76$ \\
\hline $79 / 80$ & $02 / 03$ & & $15 / 16$ & $84 / 85$ & & $88 / 89$ \\
\hline $04 / 05$ & $09 / 10$ & & & $00 / 01$ & & $98 / 99$ \\
\hline $06 / 07$ & & & & $05 / 06$ & & $99 / 00$ \\
\hline $14 / 15$ & & & & $08 / 09$ & & $07 / 08$ \\
\hline
\end{tabular}

Fonte: Organização do Autor, 2018

A classificação dos anos de Dipolo positivo ou negativo sobre o Atlântico (Tabela 4) baseou-se no cálculo do índice proposto por Servain (1991), que consiste na diferença entre as médias da TSM na área delimitada na bacia norte do Atlântico, menos a área na bacia sul (SENA, 2015). Foram considerados anos de ocorrência do Dipolo positivo aqueles anos em que o índice apresentou valor acima de $0,5^{\circ} \mathrm{C}$ e negativo os valores abaixo de $-0,5^{\circ} \mathrm{C}$, durante pelo menos dois meses consecutivos entre os meses de janeiro a junho (LUCENA, 2008).

Tabela 4 - Classificação dos anos de Dipolo positivo e negativo

\begin{tabular}{c|c}
\hline Dipolo & Anos \\
\hline \multirow{2}{*}{ Positivo } & $79 ; 80 ; 81 ; 83 ; 92 ; 97 ; 02 ; 04 ; 05 ; 10 ; 12$ \\
\hline Negativo & $84 ; 85 ; 86 ; 89 ; 91 ; 94 ; 08 ; 09 ; 14 ; 15$ \\
\hline
\end{tabular}

Fonte: Organização do Autor, 2018

\section{Resultados e discussões}

De acordo com os dados de precipitação e os cálculos realizados, a média de precipitação anual para o período de 1974 a 2016 é de 758,3 mm. Dos 43 anos analisados, 21 anos apresentam precipitação acima da média e 22 anos abaixo (Figura 2).

Ao longo desse período, a série apresentou 7 anos com precipitação total acima do limite superior, levando em consideração o desvio padrão, sendo estes: 1974, 1985, 1989, 1996, 2004, 2006 e 2011. Em contraposição, os anos de 1982, 1987, 1993, 1998, 2003, 2012, 2015 e 2016 apresentaram volume de chuva abaixo do limite inferior ao desvio padrão da série. Verifica-se que $65,12 \%$ dos anos encontram-se entre os desvios padrão, sendo assim, pode-se afirmar que são anos habituais, ou seja, encontram-se dentro da variabilidade esperada para o município. 
Figura 2 - Variabilidade interanual da precipitação para Mauriti/CE no período de 1974-2016. (As linhas horizontais na cor preta correspondem ao desvio padrão $(\square=\mathbf{2 6 0 , 8} \mathbf{m m})$, na cor vermelha identifica a média pluviométrica $(X=758,3 \mathrm{~mm}))$

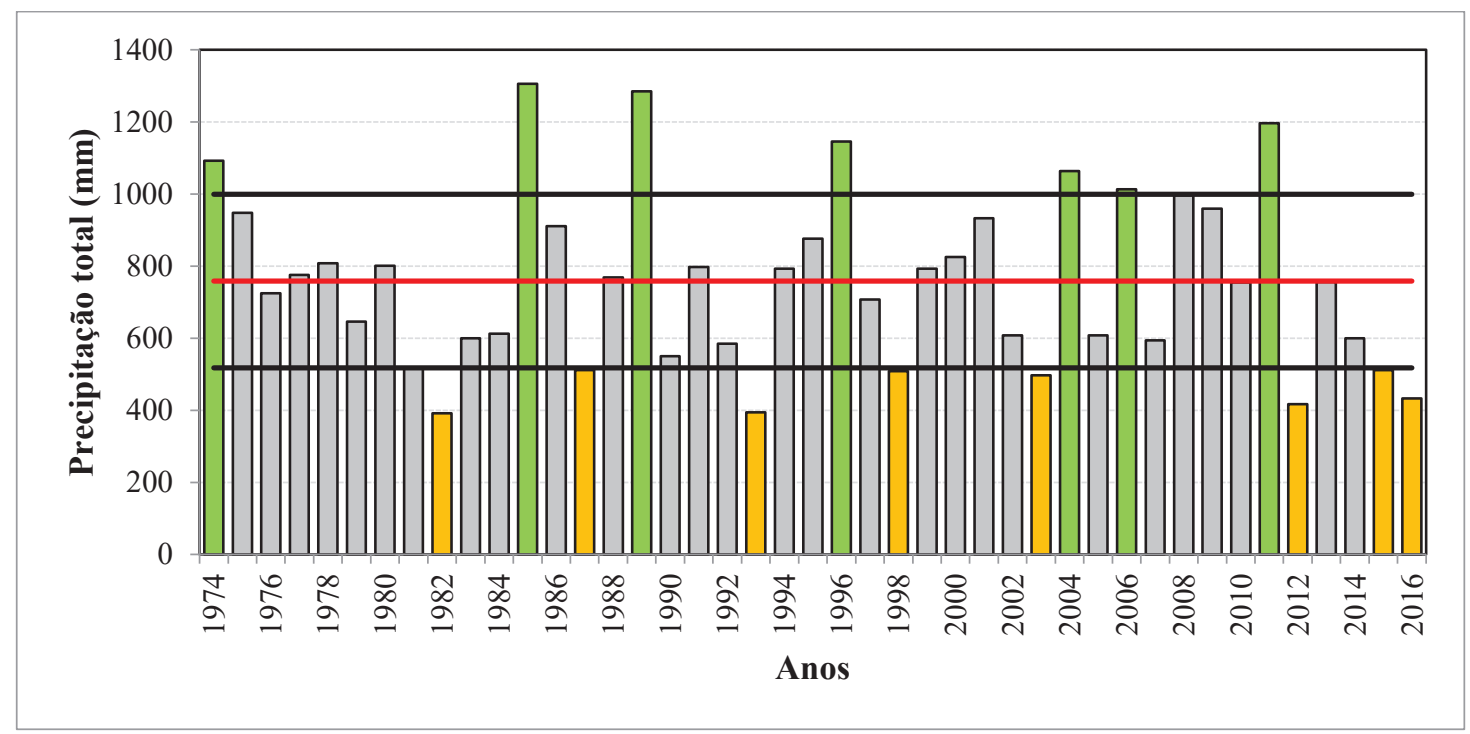

Fonte: Organização do Autor, 2018

Ao analisar o comportamento histórico das chuvas ao longo do ano - ciclo anual (Figura 3), tem-se que o período chuvoso inicia em janeiro e se estende até abril, o que corrobora com resultados encontrados por Costa e Rodrigues (2017) para Bacia do Rio Salgado, que abrange a área de estudo do presente trabalho. O mês de março apresentou o valor máximo de precipitação, com um total médio de $190 \mathrm{~mm} / \mathrm{mês}$. O quadrimestre chuvoso (JFMA) soma um volume de 596,7 mm, o que corresponde a $77,5 \%$ da precipitação total anual. O período seco está compreendido entre os meses de junho e setembro, sendo o mês de agosto o mais seco, com total médio de $1,9 \mathrm{~mm}$.

Figura 3 - Variabilidade anual da precipitação para Mauriti/CE no período de 1974-2016

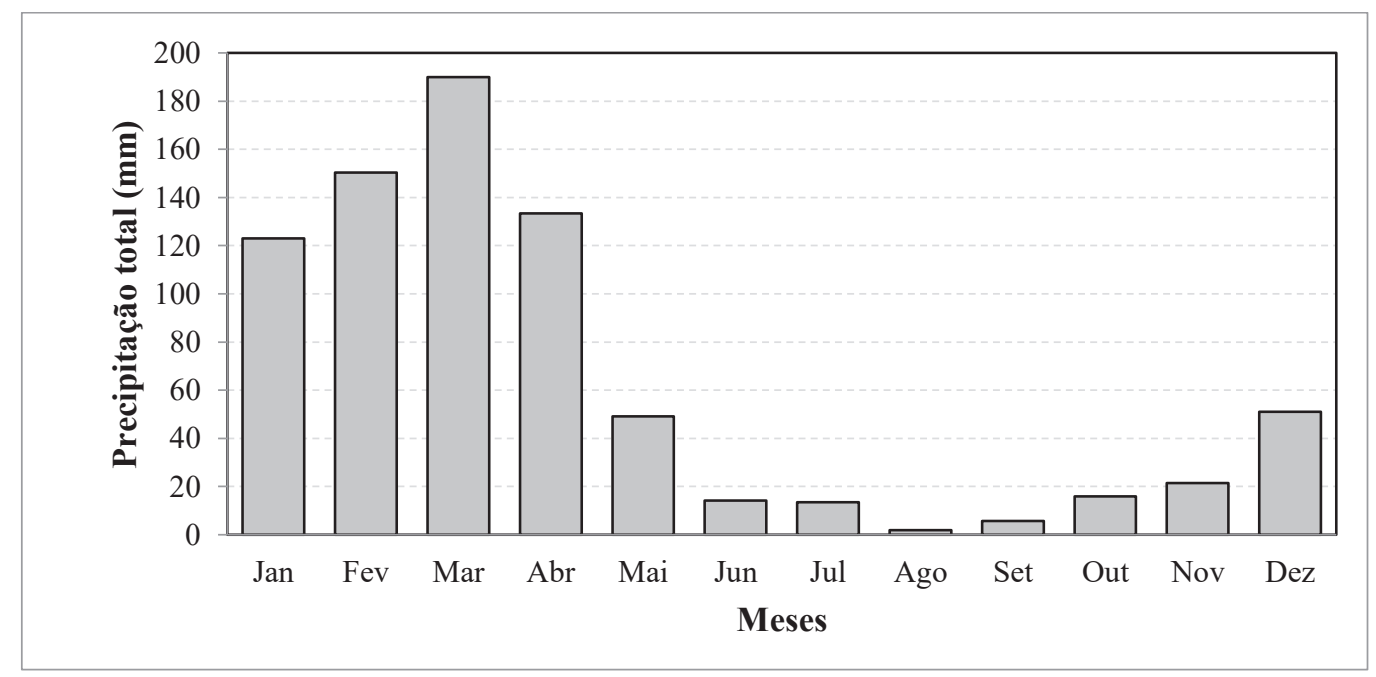

Fonte: Organização do Autor, 2018

Nos resultados para o IAC o índice positivo indica a ocorrência de um ano em que a precipitação total foi maior que a média da precipitação anual da série de dados, ou seja, um ano mais chuvoso ou úmido, enquanto que o índice negativo referese ao comportamento oposto. Na Figura 4, é perceptível que anos secos consecutivos ocorrem com uma certa frequência, como por exemplo, nos períodos de 1981-1984 e 2012-2016. 
Figura 4 - Índice de Anomalia de Chuva (IAC) para a série de dados de precipitação para Mauriti/CE no período de 1974-2016

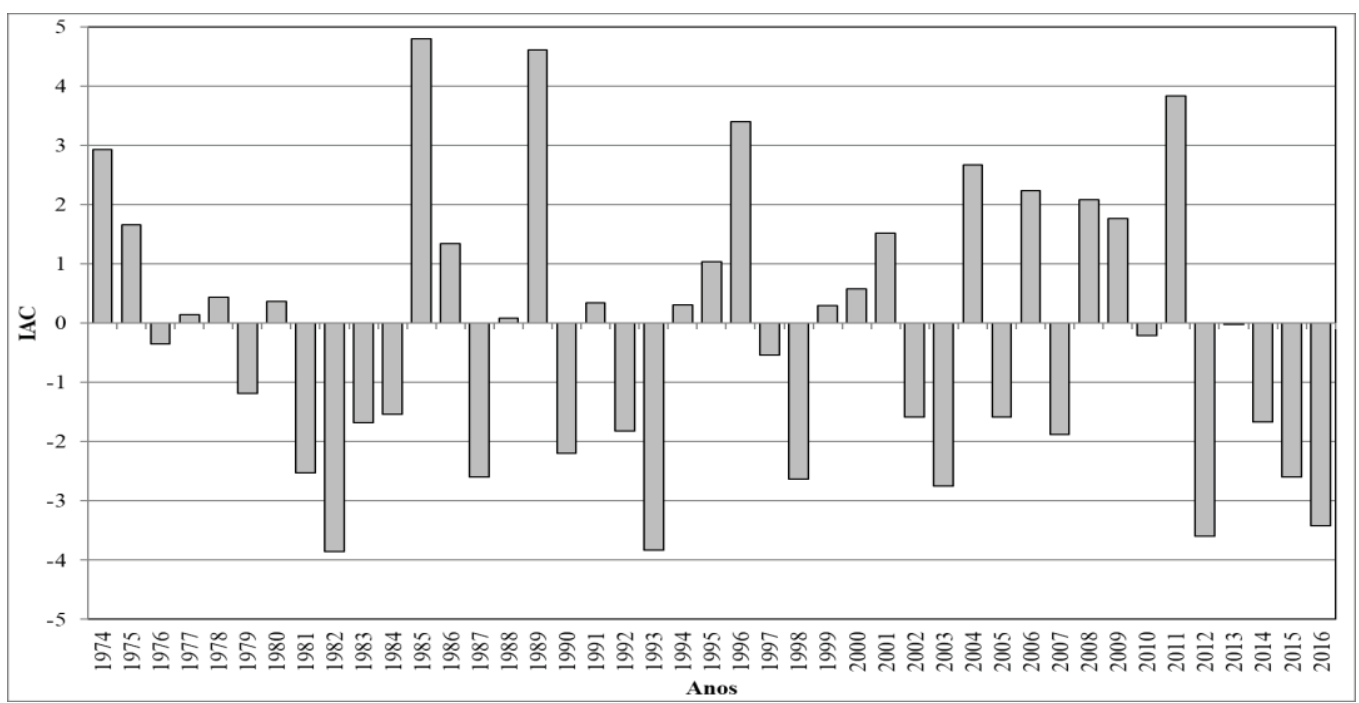

Fonte: Organização do Autor, 2018

De acordo com a classificação para os valores positivos do IAC (Tabela 1), dos 43 anos analisados, 13 anos estão inseridos na categoria "chuvoso" e 12 anos na categoria "seco", ou seja, 58,14\% dos anos analisados apresentam uma variabilidade natural (-2 < IAC < 2), apesar do aumento ou diminuição das chuvas. Os anos de 1974, 1996, 2004, 2006, 2008 e 2011 são classificados como muito chuvosos, e os anos de 1985 e 1989 foram classificados como extremamente chuvosos, apresentando, respectivamente, precipitação total de 72,2\% e 69,4\% acima da média de precipitação anual da série histórica. Com base na classificação para os valores negativos, os anos de 1981, 1982, 1987, 1990, 1993, 1998, $2003,2012,2015$ e 2016 pertencem à classe de anos muito secos. Destes anos, 1982 e 1993 corresponderem aos menores totais anuais de precipitação da série histórica, respectivamente, 48,4\% e 48\% abaixo da média climatológica. Nenhum dos anos analisados, de acordo com o IAC, foi classificado na categoria extremamente seco.

A fim de compreender as possíveis causas dos eventos extremos de precipitação na área em estudo, tornou-se necessário analisar a influência que os eventos climáticos sobre os oceanos Pacífico e Atlântico exercem sobre esses acontecimentos, tendo em vista que, como apontado anteriormente, esses eventos têm influência nos totais pluviométricos do Estado do Ceará. Os anos analisados foram selecionados de acordo com as classes de intensidade de anomalias, sendo assim, foram selecionados 2 anos extremamente chuvosos (1985 e 1989), 6 anos classificados como muito chuvosos (1974, 1996, 2004, 2006, 2008 e 2011), e os 10 anos pertencentes à classe de anos muito secos (1981, 1982, 1987, 1990, 1993, 1998, 2003, 2012, 2015 e 2016). A Tabela 5 traz a contabilização dos anos que ocorreram eventos extremos de precipitação na área em estudo, divididos de acordo com a classificação da intensidade de anomalias e a ocorrência simultânea com os eventos climáticos sobre os Oceanos Pacífico e Atlântico.

Tabela 5 - Influência dos Oceanos Pacífico e Atlântico nos eventos extremos de precipitação em Mauriti - CE (1974-2016)

\begin{tabular}{c|c|c|c|c}
\hline \multicolumn{2}{c|}{ Eventos sobre os Oceanos } & \multicolumn{3}{|c}{ Eventos extremos de precipitação } \\
\hline Pacífico & Atlântico & Extremamente chuvoso & Muito chuvoso & Muito seco \\
\hline \multirow{4}{*}{ EN } & DIPOLO + & & $04 / 05$ & $14 / 15$ \\
\cline { 2 - 5 } & DIPOLO - & & & $82 / 83 ; 87 / 88 ; 97 / 98 ; 02 / 03 ; 15 / 16$ \\
\cline { 2 - 5 } & NEUTRO & & & $11 / 12$ \\
\hline \multirow{4}{*}{ LN } & DIPOLO + & & $74 / 75 ; 07 / 08$ & \\
\cline { 2 - 5 } & DIPOLO - & $84 / 85 ; 88 / 89$ & $95 / 96 ; 05 / 06 ; 10 / 11$ & \\
\cline { 2 - 5 } & NEUTRO & & & 81 \\
\hline \multirow{3}{*}{ Neutro } & DIPOLO + & & & \\
\cline { 2 - 5 } & DIPOLO - & & & \\
\cline { 2 - 5 } & NEUTRO & & & \\
\hline
\end{tabular}

Fonte: Organização do Autor, 2018 
Os anos 1985 e 1989, classificados como extremamente chuvosos, e os anos 1974 e 2008, classificados como muito chuvosos, coincidiram com a ocorrência simultânea do fenômeno Lã Nina e o dipolo negativo do Atlântico, que segundo Ferreira e Mello (2005), são responsáveis por anos considerados normais, chuvosos ou muito chuvosos na região Nordeste do Brasil. Os anos 1996, 2006 e 2011, classificados como muito chuvosos, também coincidiram com a ocorrência do fenômeno La Niña, no entanto, para esses anos, o Atlântico apresentou condições neutras.

Embora tenha sido classificado como muito chuvoso, o ano de 2004 não coincidiu com a ocorrência do fenômeno que, segundo a literatura, geralmente a provoca, o que foi observado foi a presença do El Niño de intensidade fraca e o Dipolo positivo. Estudos como o de Giannini et al. (2004) mostram que a ocorrência concomitante desses fenômenos não favorece as chuvas no Nordeste Brasileiro.

Dos 10 anos muito secos, 6 coincidiram com a ocorrência do fenômeno El Niño, com intensidade variando entre fraco a muito forte, e apenas 1 ano coincidiu com a ocorrência do fenômeno La Niña. Em relação às condições do Oceano Atlântico, 2 anos coincidiram com o Dipolo positivo e apenas 1 ano coincidiu com a fase negativa. Os anos de 1990 e 1993, classificados como muito secos, foram anos de condições neutras para os dois oceanos, dessa forma, outros fenômenos podem ter atuado para provocar uma precipitação anual tão baixa.

No ano de 2015, classificado como muito seco, a fase negativa do Dipolo do Atlântico ocorreu simultaneamente ao El Niño de intensidade muito forte, o que demonstra que as condições do Pacífico se sobressaíram em relação às do Atlântico.

O ano de 1982 apresentou o menor total anual da série histórica $(391,5 \mathrm{~mm})$, esse ano coincidiu com condições neutras do Oceano Atlântico, no entanto, o que pode ter contribuído para uma precipitação tão baixa foi o fato do El Niño/Oscilação de Sul de 1982 - 1983 ter sido um dos mais intensos desde o início das medições, com anomalias da TSM chegando a até $4^{\circ}$ C e causando proporções catastróficas em toda a região semiárida do Nordeste (MARENGO et al., 2011).

Mesmo sendo ano de La Niña, 2012 foi classificado como muito seco, sendo assim, não seguiu o padrão indicado em vários estudos para o Nordeste Brasileiro (NEB). Embora a maioria dos eventos de seca no NEB seja atribuída ao ENOS, alguns desses eventos são consequências da posição anormalmente mais ao norte da Zona de Convergência Intertropical (ZCIT) sobre o setor do Atlântico, devido a um Oceano Atlântico Tropical Norte mais quente (Moura e Shukla 1981; Hastenrath 1990, 2012, Andreoli et al. 2012, Nobre e Shukla 1996, Marengo et al. 2013, 2016). Uma das justificativas para o que aconteceu em 2012, ano em que a La Niña fraca ocorreu simultaneamente à fase positiva do Dipolo do Atlântico.

\section{Conclusões}

O município de Mauriti apresentou média anual de $758,3 \mathrm{~mm}$ e desvio padrão de $260,8 \mathrm{~mm}$. Durante o período de 1974-2016 o ano com maior total anual de precipitação foi no ano de 1985, com 72,2\% acima da média, enquanto que 1982 apresentou o menor total anual, com apenas $391,5 \mathrm{~mm}, 48,4 \%$ abaixo da média. Verificou-se que $65,12 \%$ dos anos foram habituais, ou seja, encontram-se dentro da variabilidade esperada para o município.

Foi observado que a quantidade de anos muito secos e muito chuvosos são quase iguais. Analisando as categorias mais extremas, não foi observado nenhum ano extremamente seco, em contrapartida, foram identificados dois anos extremamente chuvosos (1985 e 1989).

Em relação a influência dos oceanos Pacífico e Atlântico, os resultados indicaram que os anos extremos apresentam um grau de relação maior com os eventos sobre o Pacífico (El Niño e La Niña) do que com Dipolo do Atlântico. Sendo observado a diminuição das chuvas na maioria dos anos com ocorrência do fenômeno EN e o aumento dos volumes pluviométricos em anos de LN. Contudo, é necessário estudos em escalas menores do que a anual, como por exemplo em escala mensal para verificar a influência mais detalhada destes fenômenos sobre a precipitação em Mauriti.

\section{References}

ALVES, et al. Índice de anomalia de chuva para diferentes mesorregiões do estado de pernambuco. Pensar Acadêmico, Manhuaçu, v. 14, n. 1, p. 37-47, janeiro-junho, 2016.

ANDREOLI, Rita Valéria; KAYANO, Mary Toshie. A importância relativa do atlântico tropical sul e pacífico leste na variabilidade de precipitação do Nordeste do Brasil. Revista brasileira de meteorologia, São paulo, v. 22, n. 1, p. 63-74, 2007. 
ARAUJO, L. E.; MORAES NETO, J.M.; SOUSA, F.A.S. Classificação da precipitação anual e da quadra chuvosa da bacia do rio Paraíba utilizando Índices de Anomalia de Chuva (IAC). Revista Ambiente \& Água - an Interdisciplinary Journal of Applied Science, v.4, n.3, 2009

CARVALHO et al. Compartimentação geoambiental da mesorregião do sul cearense, Anais XIII Simpósio Brasileiro de Sensoriamento Remoto, Florianópolis, Brasil, 21-26 abril 2007, INPE, p. 3797-3803.

CORRÊA, Irene Cristina Pereira. Análise das variabilidades interanuais e interdecenais dos índices de aridez e efeitvo de umidade do estado do Ceará. 101 p. Dissertação (Mestrado em Meteorologia) - Universidade Federal de Campina Grande, Campina Grande - PB, 2016.

COSTA, J.A.; SILVA, D.F. Distribuição espaço-temporal do índice de anomalia de chuva para o estado do ceará. Revista brasileira de geografia física, v. 10, n. 4, p. 1002-1013, 2017.

COSTA, Juliana Alcântara; RODRIGUES, Gláuber Pontes. Space-time distribution of rainfall anomaly index (RAI) for the Salgado Basin, Ceará State - Brazil. Ciência e Natura, Santa Maria, v. 39, n. 3, p. 627-634, set./dez. 2017.

CPTEC/INPE. Fim do fenômeno la niña no pacífico equatorial. Disponível em: <http://enos.cptec.inpe.br/>. Acesso em: 01 abr. 2018.

EMATERCE: SEMEANDO TECNOLOGIA NO CAMPO. Ematerce discute Fundação de cooperativa, em comunidade de mauriti-ce . Disponível em: < http://www.ematerce.ce.gov.br/index.php/noticias/14-lista-de-noticias/3985-ematercediscute-fundacao-de-cooperativa-em-comunidade-de-mauriti-ce >. Acesso em: 03 abr. 2018

FERNANDES, D. S. (et al.). Índices para a quantificação da seca. Santo Antônio de Goiás: Embrapa Arroz e Feijão, 2009. 48 p. Documentos/Embrapa Arroz e Feijão, ISSN 1678-9644; 244.

FERREIRA, Antonio Geraldo; MELLO, Namir Giovanni Da Silva. Principais sistemas atmosféricos atuantes sobre a região nordeste do brasil e a influência dos oceanos pacífico e atlântico no clima da região. Revista brasileira de climatologia, v. 1, n.1, p. 15-28, dez. 2015.

FREITAS, M. A. S. (2005). Um Sistema de Suporte à Decisão para o Monitoramento de Secas Meteorológicas em Regiões SemiÁridas. Revista Tecnologia, Fortaleza, v. Suplem, p. 84-95.

FUNCEME . Sistemas meteorológicos causadores de chuva na região nordeste do brasil. Disponível em: http:// www.funceme.br/produtos/script/chuvas/grafico_chuvas_postos_pluviometricos/entender/entender2.htm. Acesso em: 05 abr. 2018.

GIANNINI, A.; SARAVANAN, R.; CHANG, P. The preconditioning role of tropical Atlantic variability in the development of the ENSO teleconnection: implications for the prediction of Nordeste rainfall. Climate Dynamics, v. 22, p. 839-855, May. 2004.

IBGE. IBGE cidades . Disponível em: https://cidades.ibge.gov.br/brasil/ce/mauriti/panorama . Acesso em: 24 jan. 2018

IPECE. Perfil básico municipal - Mauriti. Fortaleza: IPECE, 2016. Disponível em: http://www.ipece.ce.gov.br/ perfil_basico_municipal/2016/Mauriti.pdf Acesso: Setembro de 2017.

LUCENA, D.B. Impactos dos Oceanos Pacífico e Atlântico no Clima do Nordeste do Brasil. 225 p. Tese (Doutora em Meteorologia) - Universidade Federal de Campina Grande, Campina Grande - PB, 2008.

MANIÇOBA et al., 2017. Índice de anomalias de chuva para diferentes mesorregiões do Estado do Rio Grande do Norte. Revista Brasileira de Geografia Física v.10, n.04 (2017) 1110-1119.

MARENGO, J. A.; ALVES, L. M.; BESERRA, E. A.; LACERDA, F. F. Variabilidade e mudanças climáticas no semiárido brasileiro. In: MEDEIROS, S. S.; GHEYI, H. R.; GALVÃO, C. O.; PAZ, V. P. da S. (Eds.). Recursos hídricos em regiões áridas e semiáridas. Campina Grande, INSA, 383-422, 2011. 
MARENGO, J.A.; SCHAEFFER, R.; PINTO, H.S.; ZEE, D.M.W. Mudanças climáticas e eventos extremos no Brasil. Rio de Janeiro: FBDS, 2009.

MASTER. Zona de convergência intertropical. Disponível em: <http://master.iag.usp.br/pr/ensino/sinotica/aula15/>. Acesso em: 01 abr. 2018.

MOURA, A. D., e J. SHUKLA. On the dynamics of droughts in northeast Brazil: Observations, theory and numerical experiments with a general circulation model. J. Atmos. Sci., 38, 2653 2675, 1981.

NÓBREGA, J. N. da et al. Eventos extremos de precipitação nas mesorregiões da paraíba e suas relações com a tsm dos oceanos tropicais. Revista Brasileira de Meteorologia, v.29, n.2, 197 - 208, 2014.

NOBREGA, R. S.; SANTIAGO, G. A. C. Tendência de temperatura na superfície do mar nos oceanos atlântico e pacífico e variabilidade de precipitação em Pernambuco. Mercator, Fortaleza, v. 13, n. 1, p. 107-118, jan./abr. 2014

RAMIRES, Jéssica; BERAYARMOND, Núbia; SALGADO, Carla Maciel. A VARIABILIDADE PLUVIOMÉTRICA NO CARIRI CEARENSE E A INFLUÊNCIA DAS TELECONEXÕES ENOS E ODP. In: Simpósio Brasileiro de Geografia Física Aplicada, 8., 2017, Campinas. Anais

REBOITA, Michelle Simões; SANTOS, Isimar De Azevedo. Influência de alguns padrões de teleconexão na precipitação no norte e nordeste do brasil. Revista brasileira de climatologia,[SL]v. 15,n. 10,p. 28-48,jul./dez. 2014

ROOY, M. P. VAN. A Rainfall Anomaly Index Independent of Time and Space. Notes, v.14, p. 43, 1965.

SANCHES et al., O índice de anomalia de chuva (iac) na avaliação das precipitações anuais em Alegrete/RS (19282009). Caminhos de Geografia, Uberlândia v. 15, n. 51 Set/2014 p. 73-84

SANTOS, Carlos Antônio Costa dos; MANZI, Antonio Ocimar, Eventos extremos de precipitação no estado do Ceará e suas relações com a temperatura dos oceanos tropicais. Revista Brasileira de Meteorologia, v.26, n.1, 157 $-165,2011$.

SÃO PAULO. Secretaria do Meio Ambiente. Diretrizes para a política ambiental do Estado de São Paulo. São Paulo, 1993.

SASAKI, DALTON K. Mudança nos modos de variabilidade do Atlântico Tropical no Século XX. 80 f. Dissertação (Mestrado em Ciências) - Universidade de São Paulo, São Paulo. 2014

SENA, Jaricélia Patrícia de Oliveira. Caracterização de eventos extremos de precipitação para o Cariri Paraibano e a relação com fenômenos sobre os oceanos. 55 p. Trabalho de Conclusão de Curso - Unidade acadêmica de tecnologia do desenvolvimento, Universidade Federal de Campina Grande, Sumé - PB, 2015.

SERVAIN J. Simple climatic indexes for the tropical Atlantic-Ocean and some applications. Journal of Geophysical Research - Oceans, v. 96, n. C8, p. 15137-15146, Aug. 1991.

SILVA, A. R. et al. Variações no índice de anomalia de chuva no semiárido. Journal of environmental analysis and progress, v. 2, n. 4, p. 377-384, 2017.

SOUSA, A. J. S. Eventos extremos de precipitação o leste da Amazônia. 2010. Dissertação (Mestrado) - Instituto de Ciências Atmosféricas, Universidade Federal de Alagoas, Maceió. 